hearts (ABO-compatible or -incompatible) were rejected by a purely cellular mechanism, it may be that a similar situation exists with regard to baboon-to-man transplants, and it may be possible to identify those donor-recipient pairs by the in vitro techniques used by the Johannesburg group. It could reasonably be predicted that organ survival in these selected pairs would be greatly prolonged by the pharmacologic agents currently available.

There is one other important aspect that requires clarification. In the event of rejection of a baboon organ in man, do antibodies develop that preclude retransplantation with a human organ? The limited data available with regard to this point are inconclusive.

I look forward to reports of further experimental work from the Johannesburg group in this most interesting field.

\author{
D.K.C. COOPER \\ Oklahoma Transplantation Institute \\ Baptist Medical Center \\ Oklahoma City, Oklahoma 73112
}

\section{REFERENCES}

1. Cooper DKC, Human PA, Rose AG, et al. The role of ABO blood group compatibility in heart transplantation between closely related animal species: an experimental study using the vervet monkey-to-baboon cardiac xenograft model. $J$ Thorac Cardiovasc Surg 1989; 97: 447.

2. Cooper DKC, Human PA, Rose AG. Can cardiac allografts and xenografts be transplanted across the $\mathrm{ABO}$ blood group barrier? Transplant Proc 1989; 21: 549.

3. Reichenspurner H, Human PA, Boehm DH, et al. Optimalization of immunosuppression after xenogeneic heart transplantation in primates. J Heart Transplant 1989; 8: 200.

4. Sadeghi AM, Robbins RC, Smith CR, et al. Cardiac xenograft survival in baboons treated with cyclosporine in combination with conventional immunosuppression. Transplant Proc 1987; 19: 1149 .

5. Sadeghi AM, Robbins RC, Smith CR, et al. Cardiac xenotransplantation in primates. J Thorac Cardiovasc Surg 1987; 93: 809.

6. Panza A, Roslin MS, Coons M, et al. One-year survival of heterotopic heart primate xenografts treated with total lymphoid irradiation and cyclosporine. Transplant Proc 1991; 23: 483.

\title{
THE INFLUENCE OF HLA-A2 SUBTYPE MISMATCH ON RENAL ALLOGRAFT SURVIVAL
}

Recently Fleischhauer et al. (1) described the presence of HLA-B44 subtype-specific cytotoxic T lymphocytes in a patient who rejected an HLA-matched unrelated marrow graft. We have analyzed to what extent the presence of an HLA-A2 subtype in donors or recipients of HLA-A, -B-identical or compatible cadaveric renal allograft may influence the HLAmatched kidney graft rejection.

Previously, we reported that the HLA-A2 antigen can be subdivided into four subtypes by means of cell-mediated lympholysis and biochemical analysis on isoelectric focusing gels (2-4). Population studies indicated that $89 \%$ of the HLA-A2seropositive individuals have the A2.1 major subtype, the remaining $11 \%$ may be subdivided into three $\mathrm{A} 2$ minor subtypes: $-\mathrm{A} 2.2,-\mathrm{A} 2.3$, and -A2.4 (2-4).

We have investigated whether the presence of HLA-A2 antigenic subtypes in recipients or donors of a renal allograft might have an influence on the survival of renal allografts. Of the 3000 HLA-A2-positive, HLA-A, -B-identical or -compatible combinations transplanted, a total of 51 donors and 18 recipients could be tested in the rejector group and 81 donors and 41 recipients in the control (i.e., non-rejector) group. Because it was impossible to obtain HLA-A and -B-matched HLA-A2-positive donor/recipient pairs, the HLA-A2 subtype frequencies were determined and compared between the group of non-rejected transplants and the rejected ones. The results

${ }^{1}$ This work was supported in part by the J.A. Cohen Institute for Radiopathology and Radiation Protection (IRS), the Dutch Kidney Foundation, the Eurctransplant Foundation, and the Kuratorium fur Heimdialyse. of the analyses of the A2 subtype distributions assigned by CML and biochemical analyses are summarized in Table 1 . A subdivision of the recipients into rejectors and nonrejectors revealed no significant difference. However, a subdivision of the kıdney donors into those whose kidneys were rejected and those whose kidneys functioned for at least 2 years revealed that rejectors had an HLA-A2 minor subtype significantly more often (exact $P-0.032$ ).

The strong beneficial effect of HLA-A, -B and -DR matching and effective immunosuppression makes it extremely difficult to demonstrate the significance of other factors that can increase overall renal allograft survival. Our results, which substantiate the hypothesis put forward by Fleischhauer et al. (1), suggest that HLA-A2 subtypes may exert an influence on the survival of renal allografts. When the influence of subtypes in other HLA antigens with known subdivisions-such as the HLA-A1, -A3, -B7, -B27, -B35, and -B44 antigens-is investigated, the effect of HLA subtype antigens on graft survival may be unambiguously established.

Acknowledgments. We thank Dr. Bockhorn, Dr. Donckerwolcke, Dr. Goldmann, Prof. Grabensee, Prof. Jahnke, Dr. Jansen, Prof. Koene, Prof. Land, Prof. Lange, Dr. Meider, Prof. Paul, Prof. Pichlmayr, Dr. Tegzess, Dr. Van Hooff, Dr. Weimar, Dr. Wilmink, and their nursing and administrative staffs for the generous supply of patients' cells; Dr. A. Termijtelen for critical reading; and Ms. Mulder and Mrs. Curiel for typing the manuscript.

\section{ELS GOULMY ${ }^{2,3}$ \\ JAN J. VAN DER POEL ${ }^{4}$ MARIUS GIPHART $^{2}$}

TABLE 1. HLA-A2 subtype distributions for renal allograft recipients and their donors ${ }^{a}$

\begin{tabular}{lcccccc}
\hline & Major A2 & Minor A2 & & Major A2 & Minor A2 & $P$ (exact) \\
\hline Recipients-rejector group & 18 & 0 & Recipients-nonrejector group & 40 & 1 & 0.695 \\
Donors-rejector group & 46 & 5 & Donors-nonrejector group & 80 & 1 & 0.032 \\
\hline
\end{tabular}

\footnotetext{
a Major A2 type $=$ the A2.1 subtype; Minor A2 type $=$ the A2.2 and A2.4 subtypes; $P$ is for the difference in the rejectors vs. nonrejector
} groups. 
Jos Pool ${ }^{2}$

GuIdo G. PERSIJN ${ }^{5}$

JON J. VAN ROOD ${ }^{5,6}$

JOE D'AMARO ${ }^{2}$

Department of Immunohaematology and Blood Bank

Eurotransplant Foundation

Europdonor Foundation

University Hospital

Leiden

Department of Animal Breeding

Zodiac, Wageningen, The Netherlands

${ }^{2}$ Department of Immunohaematology and Blood Bank, University Hospital, Leiden, The Netherlands.

${ }^{3}$ To whom correspondence should be addressed.

${ }^{4}$ Department of Animal Breeding, Zodiac, Marykeweg 40, Wageningen, The Netherlands.

${ }^{5}$ Eurotransplant Foundation, University Hospital, Leiden, The Netherlands.
${ }^{6}$ Europdonor Foundation, University Hospital, Leiden, The Net erlands.

\section{REFERENCES}

1. Flesschhauer K, Kernan N, O'Reilly RJ, Dupont B, Yang SY. Bor marrow allograft rejection by $\mathrm{T}$ lymphocytes recognizing a sing amino acid difference in HLA-B44. N Engl J Med 1990; 32 1818.

2. Horai S, Van der Poel JJ, Goulmy E. Differential recognition of t serologically defined HLA-A2 antigen by allogeneic cytotoxic cells: I. Population studies. Immunogenetics 1982; 16: 135.

3. Van der Poel JJ, Pool J, Goulmy E, Van Rood JJ. Different1 recognition of the serologically defined HLA-A2 antigen by a logeneic cytotoxic T cells: II. Definition of three HLA-A2 sul types by CTLs. Immunogenetics 1983; 17: 599.

4. Van der Poel JJ, Molders H, Thompson AJ, Ploegh HL. Definitio of four HLA-A2 subtypes by CML typing and biochemical anal. sis. Immunogenetics 1983; 17: 609.

\section{HUMAN HERPESVIRUS-6 INFECTION AND RENAL TRANSPLANTATION}

Human herpesvirus-6 (HHV-6) infection has been recently reported in renal transplant patients $(1,2)$. In our study HHV6 infection was serologically investigated by indirect immunofluorescence (Stellar Bio System IFA, Columbia, MD) in 53 renal transplant patients ( 39 male and 14 female) aged 12 to 64 years. All of them were under triple therapy (cyclosporine, azathioprine, and prednisone), and if acute kidney rejection occurred they were given methylprednisolone following the protocol previously described (3).

Before transplantation 40 patients (75.5\%) were HHV-6seropositive, according to data reported by Okuno et al. (2), and no correlation with age, sex, and time on dialysis was found. Antibody titers ranged from 1:20 to $1: 1280$, and in 31 patients $(77.5 \%)$ were less than or equal to $1: 80$.

After transplantation 10 seronegative patients (77\%) had anti-HHV-6 seroconversion. Seven of them had received a kidney from an HHV-6-seropositive donor and 1 from a seronegative donor; for the remaining 2 recipients the HHV-6 serological status of donors was unknown. Eight seroconversions $(80 \%)$-in particular 5 of the 7 from seropositive donorsoccurred during the first month.

Twenty-five seropositive patients $(62.5 \%)$ had a significant increase in antibody titer as well. Eighteen of them had received a kidrey from an HHV-6 seropositive donor and 4 from a seroregative donor; for the remaining 3 recipients the HHV- 6 serological status of the donor was unknown. In 23 of these patients (92\%)--in particular in all 18 from seropositive donors - the titer increase occurred during the first month; in 19 (76\%) antibody titers were $>1: 320$. This frequency of active HHV-6 infection after transplantation is higher than frequencies reported by Okuno et al. (2) and lower than those found by Morris et al. (1) (66\% vs. $38 \%$ and $82 \%$, respectively).

Transmission of HHV- 6 infection by transplanted organs is open to discussion $(1,4,5)$. We observed that all seronegative patients who received a kidney from a seropositive donor had HHV-6 seroconversion; furthermore, 11/13 $(84.6 \%)$ pairs of patients who received a kidney from the same seropositive donor reacted in the same way: in 2 pairs neither had any infection and in 9 pairs both had active HHV- 6 infection. Only in the remaining 2 pairs of patients did the members react in a different way: one had HHV-6 active infection while the othe had no infection. This suggests that the transmission of th infection depends more on the degree of infectivity of th transplanted organ than on the susceptibility of the recipien as previously hypothized for CMV infection (6).

With regard to CMV infection, $48 / 53$ patients $(90.6 \%)$ wer seropositive before transplantation. After transplantation $2 /$ $(40 \%)$ seroconversions and $34 / 48(70.8 \%)$ significant increase in CMV antibody titer were observed. Both seroconversior occurred during the second month after transplantation, an $24 / 34(70.6 \%)$ of the increases in antibody titer were betwee the second and third months. These variations in CMV sero ogical status occurred later than those in HHV-6, suggestin no relation between these two infections.

Okuno et al. (2) reported a close connection between vir activation and acute kidney rejection. In our study rejectio occurred in $11(20.7 \%)$ patients, and in 8 of them $(72.7 \%)$ occurred during the first two months after transplantatios affecting 9/35 (25.7\%) patients with active HHV-6 infectio and $2 / 18(11.1 \%)$ patients without infection; notwithstandin the higher frequency of rejection in patients with an activ HHV-6 infection, no significant correlation could be demor strated $(P=0.2144)$.

\section{Chiara Merlino ${ }^{1}$ \\ Franca Giacchino ${ }^{2}$ \\ GIUSEPPE P. SEGOLONI ${ }^{2}$ \\ ALESSANDRo Negro Ponzi ${ }^{3}$ \\ Institute of Microbiology \\ Institute of Nephrology \\ University of Turin \\ Turin, Italy}

\footnotetext{
${ }^{1}$ Institute of Microbiology, University of Turin, Via Santena 9, 101 Turin, Italy.

${ }^{2}$ Institute of Nephrology
}

\section{REFERENCES}

1. Morris DJ, Path MR, Littler E, Arrand JR, Mallick NP, Johnsc $R$. Human herpesvirus -6 infection in renal-transplant recipient 